\title{
A PROPOS DE QUELQUES NOUVEAUX PARASITES ENDOGLOBULAIRES TROUVÉS EN ROUMANIE
}

\section{Par Georges IRIMINOIU}

La Roumanie est le pays de l'histoire des piroplasmoses, découvertes par Babès en 1888, dans le sang des bœufs atteints d'hém.Jglobinurie.

Par conséquent, Babès a déterminé la cause de l'hémoglohinurie des bovidés, provoquée par un parasite localisé dans le globule rouge et dénommé par lui Hæmatococus bovis et plus tard par son élève Starcovici, Babesiella bovis (1893).

En 1893, Neagu décrit dans sa thèse, avec précision, Piroplasma bigemina (Smith et Kilborne, 1893).

C'est le piroplasme qui se trouve partout dans notre pays. Nous l'avons trouvé aussi en Transylvanie en avril 1940.

Depuis ces découvertes, on n'a plus trouvé d'autres espèces de piroplasmes dans notre pays chez les bovins.

Nous avons eu l'occasion de mettre en évidence, pour la première fois, en Roumanie, dans le sang des bovins et chez les moutons, de nouveaux parasites d'origine exotique, qui sont classés parmi les sporozoaires. Ceux-ci sont les suivants, dans l'ordre de leur découverte :

\section{Parasites}

1. Babesiella major, en avril $1937 ; 2$. Theileria parva, en octobre 1942 ; 3. Anaplasma marginale chez le bouf, en avril 1947 et Anaplasma ovis, en mai $1947 ; 4$. Babesiella berbera, en avril 1947.

L'aire géographique de ces parasites est à présent seulement la Transylvanie.

Ces parasites du sang des animaux ont été bien étudiés récemment en Algérie, par Edmond Sergent, A. Donatien, L. Parrot et F. Lestoquard.

Les bovins hébergeant Babesiella major et Babesiella berbera ont presque les mêmes sympiômes : tristesse, inappétence, amaigrisse-

Ans, de Parasitologie, T, XXIII, Nos 5-6, - 1948, p. 296-300. 
ment progressif, absence de rumination, chute rapide de la sécrétion lactée. La température monte jusqu'à $41^{\circ}$ et peut être coupée de rémittences. Puis apparaissent l'ictère et l'hémoglobinurie. L'urine a une couleur bordeaux ou café.

I. Morphologiquement, la Babesiella major occupe toujours une position centrale dans les hématies.

Les éléments ronds ne diffèrent pas beaucoup des formes analogues de Babesiella berbera et de Babesiella bovis.

Les éléments qui les distinguent d'autres Babesiella sont les formes allongées subpiriformes ou en forme de citron; elles représentent un tiers du nombre total. Quand ces formes allongées sont doubles, elles forment un angle inférieur à un angle droit, caractère qui les distingue de Babesiella bovis, qui est placée à l'extrême bord du globule rouge, comme sì elle le coiffait « en calotte ». Les formes allongées sont un peu plus petites que celles du Piroplasma bigeminum.

II. Morphologiquement la Babesiella berbera a des formes anaplasmoïdes qui ne se distinguent des vrais anaplasmes que par la taille.

Les formes rondes sont les plus nombreuses et représentent les formes adultes des parasites. Elles sont placées une ou deux dans le globule rouge, avec une forme parfaitement circulaire. "Elles sont constituées par un anneau cytoplasmique entourant une vacuole centrale, d'un blanc cru, au niveau de laquelle le globule semt!le troué à l'emporte-pièce. »

A un certain nombre des formes rondes, on voit attachée une queue de cytoplasme, comparable au pétiole d'une feuille. II y a aussi des formes allongées elliptiques. On voit encore des formes en cours de division binaire.

Un élément rond s'allonge et, par un étranglement bilatéral, se divise en deux cellules-filles, unies entre elles par un filament cytoplasmique. Le filament peut disparaître et il reste deux éléments ronds adultes.

III. Les bovins hébergeant Theileria parva ont des symptômes qui se manifestent par l'hyperthermie, la tristesse, l'abattement; la tête est basse, avec les paupières tuméfiées et mi-closes. La muqueuse conjonctivale est congestionnée, puis devient blanche avec de multiples pétéchies rondes. Plus tard, elle présente une teinte nettement ictérique, comme la muqueuse buccale. 
On constate aussi un amaigrissement rapide, la perte de l'appétit et de la rumination avec une atonie du rumen. Les mouvements respiratoires et les battements cardiaques sont accélérés.

On observe encore parfois de la tuméfaction des ganglions lymphatiques préscapulaires et précruraux et quelquefois de la diarrhée sanglante et de l'hématurie. L'anémie est le symptôme caractéristique. Le sang devient clair et fluide, avec des lésions assez marquées, particulièrement de l'anisocytose et de la poïkilocytose.

A l'autopsie, les animaux qui succombent de theilériose présentent des altérations organiques remarquables: suffusions sanguines sous-épicardiques et ecchymoses dans l'endocarde. La rate est hypertrophiée. La muqueuse de la caillette et des intestins peut présenter des ecchymoses et des ulcérations. Le foie est volumineux et marbré par des hémorragies sous-capsulaires. Les ganglions lymphatiques sont également hypertrophiés et entourés d'un œè̀me gélatineux ou hémorragique. Les reins sont de même enveloppés d'un œdème gélatineux; on y constate des infarctus sous-capsulaires.

Nous avons trouvé dans les ganglions lymphatiques, des « corps en grenade " et dans le sang des globules rouges parasités par les gamétocytes. Le nombre des gamétocytes dans une hématie parasitée, varie de 3-5 jusqu'à 10 .

Le caractère qui différencie Theileria parva des autres espèces, est la morphologie des gamétocytes ; il y a prédominance des éléments en bâtonnets ou en points, contrairement à la prédominance des formes rondes ou ovalaires que l'on constate chez les autres Theileridae.

Nous n'avons pas eu l'occasion de faire l'épreuve de prémunition croisée et l'épreuve du xénodiagnostic.

IV. Les bovins et les moutons atteints d'anaplasmose ont presque les mêmes symptômes, qui se caractérisent par un amaigrissement progressif qui aboutit à une extrême cachexie. Les conjonctives peuvent devenir peu à peu blanches comme de la porcelaine. Les animaux meurent dans un état d'épuisement complet.

On ne peut observer d'ictère, ni d'hématurie, ni de diarrhée sanglante. La température est irrégulière et peut s'élever à $40^{\circ}$.

A l'autopsie, les lésions remarquables sont celles de l'anémie et de la cachexie : épanchements séreux dans les grandes cavités. Le foie est pâle et friable. La rate est augmentée de volume, avec suffusions sanguines sous-capsulaires. Les ganglions lymphatiques sont hypertrophiés et œdématiés, avec des hémorragies sous-capsulại- 
res et enveloppées d'un œdème gélatineux. Le cœur est le siège de suffusions sanguines sous-épicardiques. Les reins sont dégénérés, avec œdème gélatineux. Le sang présente des lésions assez marquées et en particulier de l'anisocytose et une diminution du nombre des hématies.

Du point de vue morphologique, l'anaplasme qui est identifiée chez les bovins comme Anaplasma marginale et chez les moutons comme Anaplasma ovis, se présente sous un seul aspect : celui d'un grain sphérique. On ne peut distinguer chez lui ni noyau, ni cytoplasme. Il se colore très bien par les couleurs basiques. Par la méthode panoptique il se colore en brun-mat avec une nuance rouge. Ces petits grains sont situés, le plus souvent, sur le bord même du globule rouge et parfois (environ $1 / 5$ ) à l'intérieur du globule. Il y a aussi des globules qui possèdent deux grains rapprochés sur le bord du même globule rouge, ou les deux grains sont situés sur les côtés opposés du bord du même globule rouge.

\section{Agents vecteurs}

Les tiques, récoltées sur des animaux atteints d'anaplasmose, ont été identifiées comme appartenant à l'espèce Dermacentor reticulatus qui transmet le virus par piqûre à d'autres animaux. Cela correspond avec les mesures de prophylaxie suivantes :

Les moutons d'un troupeau infesté par ce parasite, sont tondus et complètement débarrassés des tiques (Dermacentor reticulatus), par des bains de créoline ; puis on les change de pâturage. La maladie semble disparaître et probablement s'est éteinte. Il n'y en a aucun cas nouveau dans l'intervalle de trois mois.

Les tiques, récoltées sur les animaux malades et qui transmettent probablement le virus par piqûre à d'autres animaux, ont été identifiées par le Professeur G. Senevet, spécialiste en ixodinés. Ce sont les suivantes :

1. Moutons atteints d'anaplasmose: Dermacentor reticulatus, Hæmaphysalis punctata et un insecte pupipare, Melophagus ovinus.

2. Bovins atteints de theileriose : Dermacentor reticulatus, Hæmaphysalis punctata et Ixodes ricinus.

3. Bovins atteints de Babesiella berbera: Ixodes ricinus.

4. Bovins atteints de Babesiella major et d'Anaplasma marginale, selon notre identification : Dermacentor reticulatus et Ixodes ricinus.

Nous signalons la présence de ces parasites qui, jusqu'à présent, 
étaient inconnus dans notre pays. Des études plus amples seront probablement entreprises dans cette direction par nos spécialistes.

A cette occasion, nous transmettons nos vifs remerciements à M. le $\mathrm{D}^{\mathrm{r}}$ Edmond Sergent, Directeur de l'Institut Pasteur d'Algérie, pour son amabilité et la bienveillance qu'il nous a témoignée en confirmant l'identité des parasites : anaplasmes, Babesiella major, Babesiella berbera et au Professeur G. Senevet, pour l'identification des tiques.

\section{BiBLIOGRAPHIE}

Cernatanu (G.). - Piroplasmes et. Piroplasmoses des animaux domestiques en Roumanie. Arch. Roum. de Pathologie expér., X, n 3, sept. 1937.

Curasson. - Traité de protozoologie vétérinaire et comparée, 1943.

Traité de pathologie exotique vétérinaire et comparée, 1942.

Doflein (F.) et RFichenow (Ed.). - Lehrbuch der Protozoenkunde, 1939.

Hutyra (Marek-Manninger). - Spezielle Pathologie und Therapie der Haustiere. Iniminoru (G.). - Despre piroplasme si piroplasmosele bovideelor in Transilvania. Bul. As, Méd. Vét., n ${ }^{\circ} 1-2,1942$.

- Despre piroplasme. Ciclul evolutiv schizogonic (Theileriose). Tipografia Progresul, Sibiu, 1945.

- Despre Anaplasma marginale si Anaplasmose. Tipografia Cartea Româneasca, 1947.

- Prezenta Theilerioselor in Romània. Tip. C. Româneasca, 1947.

-.. Prezenta Anaplasmoselor in România. Anaplasma marginale si Anaplasma ovis. Agricultura, 1947.

- Aparitia Babesiellei Berbera in România. Tip. Cartea Româneasca, 1947.

Neuman (R.-O.) und Martin Mayer. - Tierische Parasiten.

Neveu-Lemare (M.). - Traité de Protozoologie médicale et vétérinaire, 1943.

- Traité d'Entomologie médicale et vétérinaire, 1938.

Schllling und MEYer (F.). - Piroplasmosen. Handbuch pathogenen Mikroorganismen, 1927.

Sergent (Ed.), Donatien (A.), Parrot (L.) et Lestoguard (F.). - Etudes sur les piroplasmoses bovines, 1945.

Sergent (Ed.). - Lettre personnelle du 30 mai 1947.

- Lettre personnelle du $1^{\text {er }}$ juillet 1947.

Laboratoire de Bactériologie vétérinaire de Cluj (Roumanie) 